\title{
Kernos
}

Revue internationale et pluridisciplinaire de religion grecque antique

26 | 2013

Varia

\section{Michel Malaise, À la découverte d'Harpocrate à travers son historiographie}

\section{Richard Veymiers}

\section{(2) OpenEdition \\ Journals}

\section{Édition électronique}

URL : http://journals.openedition.org/kernos/2174

DOI : 10.4000/kernos.2174

ISSN : 2034-7871

\section{Éditeur}

Centre international d'étude de la religion grecque antique

\section{Édition imprimée}

Date de publication : 10 octobre 2013

Pagination : 435-437

ISSN : 0776-3824

\section{Référence électronique}

Richard Veymiers, « Michel Malaise, À la découverte d'Harpocrate à travers son historiographie », Kernos [En ligne], 26 | 2013, mis en ligne le 10 octobre 2013, consulté le 02 mars 2021. URL : http:// journals.openedition.org/kernos/2174; DOI : https://doi.org/10.4000/kernos.2174

Ce document a été généré automatiquement le 2 mars 2021.

Kernos 


\title{
Michel Malaise, À la découverte d'Harpocrate à travers son historiographie
}

\author{
Richard Veymiers
}

\section{RÉFÉRENCE}

Michel Malaise, À la découverte d'Harpocrate à travers son historiographie, Bruxelles, Académie Royale de Belgique, 2011. 1 vol. $16 \times 24 \mathrm{~cm}, 167$ p. (Mémoire de la Classe des Lettres. Collection in- $8^{\circ}$, 3e série. Tome LVII, $n^{\circ} 2079$ ). ISBN : 978-2-8031-0285-3.

1 Optant pour une approche novatrice, très originale, Michel Malaise entreprend de faire découvrir l'une des figures divines les plus attachantes des panthéons égyptien et gréco-romain par le biais de son historiographie. De l'Antiquité à nos jours, nombre de

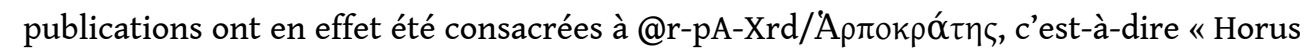
l'Enfant ", le fils posthume d'Isis et d'Osiris. Les passer en revue permet à l'A. de dresser le « portrait-robot » du petit dieu, d'en présenter les multiples facettes, tout en faisant ressortir l'évolution des réflexions et le progrès des connaissances qui s'y rapportent.

Ce sont ainsi les «méandres d'un domaine de recherche » (p. 12) évoluant rapidement et dont l'A. a marqué certaines étapes essentielles ${ }^{1}$ - qui se dessinent en filigrane à travers la trame chronologique de l'ouvrage. Sans doute n'aurait-il pas été inutile pour les lecteurs d'inscrire ces diverses études centrées sur Harpocrate dans un contexte historiographique plus large et d'expliciter ainsi davantage le cheminement ayant conduit à la naissance d'une discipline à part entière, que Jean Leclant a consacré officiellement en 1999 sous le néologisme d'《 isiacologie »", et où le territoire nilotique a repris sa juste place à l'occasion du $I V^{e}$ colloque international sur les études isiaques tenu en 2008, précisément en hommage à Michel Malaise ${ }^{3}$.

Cette historiographie harpocratique, structurée en huit périodes d'inégale durée correspondant à autant de chapitres, est le fruit d'une vaste enquête bibliographique à 
la suite de laquelle il a fallu opérer une sélection, dont les critères sont exposés dans une introduction sans doute trop concise (p.11-13). Ce sont les études parues après 1980, dont quelques-unes, majeures, signées par l'A. lui-même ${ }^{4}$, qui ont retenu prioritairement son attention, puisqu'elles couvrent près de deux tiers du volume (p. 43-120). Ce déséquilibre reflète certes l'épanouissement récent du domaine, mais les périodes antérieures auraient pu faire l'objet d'un plus large traitement. Le chapitre I (p.15-19), par exemple, offre une transition un peu abrupte entre l'Antiquité, représentée par Plutarque, et Pirro Ligorio au Cinquecento, sans évoquer saint Augustin $^{5}$, dont les écrits ont pourtant participé à assurer au Moyen Âge le souvenir du petit dieu « silencieux », posant le doigt à la bouche.

4 Les notices composant ces chapitres chronologiques sont réalisées avec le soin, la clarté et la précision qui caractérisent toute l'œuvre de l'A. On y trouve ainsi un très riche corps de notes infrapaginales, toujours fort à propos. Bien souvent, il ne se contente pas d'extraire les idées fortes offertes par une publication; il se veut aussi critique, n'hésitant pas à émettre son avis, à rectifier des erreurs de jugement ou des imprécisions. À ce titre, les notices rappellent celles qui alimentent l'Inventaire bibliographique des Isiaca (IBIS) que Jean Leclant et Gisèle Clerc avaient publié en 1972-19916 et dont la Chronique bibliographique de la Bibliotheca Isiaca est depuis 2008 le prolongement ${ }^{7}$.

5 Dans la mesure où la chronologie sert de fil conducteur, cette historiographie ne se prête pas à une lecture véritablement linéaire, laquelle fait apparaître d'inévitables répétitions. C'est un instrument de travail qui se veut avant tout consultatif. D'où l'importance d'une conclusion à visée synthétique (p. 121-133) récapitulant la matière sous des points de vue thématiques, révélant l'état actuel de la recherche avec les acquis et les perspectives.

6 Ayant fait l'objet en 2006 d'une monographie par Sandra Sandri, bien détaillée par l'A. (p. 103-109), la genèse d'Har-pa-chered, apparaît aujourd'hui plus claire. Si son théonyme remonte à la $21^{\mathrm{e}}$ et son iconographie, à la $22^{\mathrm{e}}$ dynastie, c'est Thèbes, plutôt que Coptos, qui semble bien lui avoir servi de berceau théologique. Quant aux raisons de son invention, bien que plusieurs scénarios soient possibles, elles sont vraisemblablement à chercher dans les rites de perpétuation de la légitimité royale à travers l'identification du souverain à un enfant divin.

7 Son rayonnement ne fait qu'augmenter au I ${ }^{\text {er }}$ millénaire, parallèlement à celui d'autres dieux-enfants, tels Chonsou l'Enfant et Harsomtous, dont il peut partager la même iconographie, comme l'indiquent nombre de statuettes de bronze inscrites (p. 84-85). En l'absence de légende explicite, l'identité des représentations est ainsi sujette à caution, reflet d'une polyvalence dont peinent à rendre souvent compte les étiquettes modernes, mais qui est pourtant si révélatrice de la nature du polythéisme égyptien.

Avec l'avènement des Lagides, un pendant hellénisé d'Har-pa-chered fait son apparition sous le nom d'Harpocratès dans des circonstances qui demeurent à préciser. Cette évolution, qui n'est pas qu'iconographique, n'éclipse pas pour autant la forme pharaonique. Sans doute aurait-il dès lors été pertinent de distinguer davantage ce qui relève d'Har-pa-chered et d'Harpocratès. Les deux formes ne s'inscrivent pas dans les mêmes dynamiques, n'évoluent pas toujours dans les mêmes sphères. Si l'une s'affirme davantage dans le monde officiel des temples égyptiens, l'autre concerne avant tout «le monde des images humbles » (p. 132), celui de la religion dite " populaire », c'est-à- 
dire "privée », comme l'atteste sa prédominance dans la coroplathie égyptienne, où elle se décline en une multitude de types.

9 La présence du dieu-enfant hellénisé varie ainsi fortement d'un médium à un autre, d'où l'importance, l'urgence même, de constituer des répertoires iconographiques autorisant des "comparaisons chiffrées » (p. 127) entre les supports. Très instructifs, ces écarts fluctuent selon les lieux et les époques. Le succès d'Harpocrate s'exprime ainsi hors d'Égypte surtout à travers les bronzes, les bijoux et les gemmes, alors que sa place reste très discrète dans la religion officielle, bien que les monnaies à son effigie soient plus fréquentes à partir du $\mathrm{II}^{\mathrm{e}} \mathrm{s}$. ap. J.-C.

Le tableau global demeure donc assez éclaté. Une vaste synthèse reste à écrire sur l'histoire complexe du petit dieu à l'époque gréco-romaine, en tenant compte du réseau de relations qu'il noue avec les autres membres de sa famille divine, mais aussi avec les représentants de multiples panthéons locaux. Grâce au bel outil que nous offre Michel Malaise dans cet ouvrage qui s'achève par une bibliographie complète (p. 137-152) et un index général très détaillé (p. 153-166), une telle entreprise parait aujourd'hui plus que jamais envisageable.

\section{NOTES}

1. Depuis sa thèse monumentale sur Les conditions de pénétration et de diffusion des cultes égyptiens en Italie, Leiden, 1972 (ÉPRO, 22), jusqu'à la réflexion méthodologique conduite dans son essai Pour une terminologie et une analyse des cultes isiaques, Bruxelles, 2005 (Mémoires de la Classe des Lettres de l'Académie royale de Belgique. Collection in $-8^{\circ}, 3^{\mathrm{e}}$ série, 35$)$.

2. J. Leclant, « 40 ans d'études isiaques : un bilan », in L. Bricault (éd.), De Memphis à Rome. Actes du Ier Colloque international sur les études isiaques, Poitiers - Futuroscope, 8-10 avril 1999, Leiden/Boston/ Köln, 2000 (RGRW, 140), p. xxii.

3. L. Bricault, M.J. Versluys (éd.), Isis on the Nile. Egyptian Gods in Hellenistic and Roman Egypt. Proceedings of the IVth International Conference of Isis Studies, Liège, November 27-29, 2008. M. Malaise in honorem, Leiden/ Boston, 2011 (RGRW, 171).

4. On signalera particulièrement l'article de synthèse « Harpocrate. Problèmes posés par l'étude d'un dieu égyptien à l'époque gréco-romaine ", BAB 11 (2000), p. 401-431.

5. Var., ap. August., De civitate Dei, 18, 5, repris au xiiie ${ }^{\mathrm{s}}$. dans le fameux recueil des Gesta Romanorum (éd. Oesterley, cap. 22).

6. J. Leclant, G. Clerc, Inventaire bibliographique des Isiaca (IBIS). Répertoire analytique des travaux relatifs à la diffusion des cultes isiaques 1940-1969, Leiden, 1972-1991 (ÉPRO, 18/1-4).

7. L. Bricault (éd.), Bibliotheca Isiaca I, Bordeaux, 2008, p. 131-230; L. Bricault, R. Veymiers (éd.), Bibliotheca Isiaca II, Bordeaux, 2011, p. 317-448. 


\section{AUTEURS}

\section{RICHARD VEYMIERS}

F.R.S.-FNRS - Université de Liège 